\title{
Transient global amnesia: Isolated event or healthy predictor? Clinical experience of an Italian Emergency Department
}

\author{
Greta Barbieri, Alessandro Cipriano, Simona Luly, Viola Del Nista, Eugenio Orsitto, \\ Massimo Santini
}

Department of Emergency Medicine, University Hospital of Pisa, Italy

\begin{abstract}
Transient global amnesia (TGA) is a clinical syndrome characterized by reversible anterograde amnesia, in which the patient is alert, self-awareness appears intact and other neurological symptoms are absent. The diagnosis is based on the following criteria: i) witnessed event; ii) acute onset of anterograde amnesia; iii) no accompanying neurological symptoms; iv) no alteration of consciousness; v) no epileptic features; vi) resolution within 24 hours; vii) exclusion of other causes.

We conducted a cohort study at the Department of Emergency Medicine on 119 patients with TGA diagnosis from 2010 to 2014, with follow-up evaluation by telephone interview.

The objectives of our study were to evaluate the frequency of subsequent episodes, to identify predisposing factors, and to investigate whether TGA is a possible predictor of neurological disease.

The frequency of comorbidity in our population was in line with literature. We observed a recurrence rate of $9.5 \%$, with a prevalence for the male gender, while no other factor correlates with TGA recurrence. TGA was not a predictor of further neurological diseases.
\end{abstract}

Correspondence: Greta Barbieri, Department of Emergency Medicine, University Hospital of Pisa, via Paradisa 2, 56124 Pisa, Italy.

Tel. +39.050.997827.

E-mail: greta.barbieri88@gmail.com

Key words: Amnesia; Emergency; Epilepsy; Stroke.

Contributions: GB was responsible for drafting and reviewing the manuscript; AC, MS designed the study and revised the manuscript; EO revised the manuscript; SL, VDN were responsible for data collection.

Conflict of interest: the authors declare no potential conflict of interest.

Funding: none.

Conference presentation: the work was presented as an oral communication at the AcEMC National Congress (November 7-9, 2018, Pisa, Italy).

Received for publication: 16 April 2019.

Revision received: 19 July 2019.

Accepted for publication: 17 September 2019.

This work is licensed under a Creative Commons Attribution 4.0 License (by-nc 4.0).

${ }^{\circ}$ Copyright: the Author(s), 2019

Licensee PAGEPress, Italy

Emergency Care Journal 2019; 15:8217

doi:10.4081/ecj.2019.8217
In conclusion, TGA is a benign pathology with a low probability of relapse. Accordingly, management in Emergency Department should be based on a correct initial clinical classification for rapid discharge.

\section{Introduction}

Transient global amnesia (TGA) is a clinical syndrome characterized by prominent reversible anterograde amnesia, with inability to form new memories. During the attack, the patient is alert and communicative, self-awareness appears to be intact, focal neurological and epileptic features are absent. Other cognitive functions are spared and neurologic exam is otherwise normal. ${ }^{1-3}$ The inability to form new memories is one of the main clinical features, since patients are disoriented in time and often repetitively ask questions about the date or their environment. Retrograde amnesia is common, but variable and it can extend back hours to days, weeks, and rarely years..$^{1,2}$

In the general population, the incidence of TGA has been estimated as 5.2 to 10 per 100,000 per year, without significant differences according to gender. The majority of episodes occurs in individuals between the ages of 50 and 80 years, with a mean age of onset between 60 and 62 years..$^{1,45}$

Hypotheses on TGA's pathogenesis include psychological disturbances, personality traits, ${ }^{6}$ paradoxical embolism, ${ }^{7,8}$ hypoxicischemic origin (associated with venous congestion in memory relevant structures),, 910 epilepsy, ${ }^{11}$ migraine, ${ }^{2,12}$ Cortical Spreading Depression, ${ }^{12}$ but its cause is still unknown. ${ }^{7}$ Quantitative imaging studies in TGA have shown hypoperfusion or hypometabolism in the temporal lobe, decreased activation in the temporal-limbic circuit during a scene encoding task with functional magnetic resonance imaging and changes in the CA-1 neurons of the lateral hippocampus with diffusion tensor imaging. ${ }^{13,14}$

Trigger events have been clearly associated with the attack such as physical events (strenuous activity, sexual intercourse, Valsalva maneuver, intense pain), emotional stress, postural changes, medical procedures, high altitude, contact with water, changes in body temperature..$^{15,16}$

TGA's diagnosis is still based on diagnostic criteria established by Hodges and Warlow in 1990. ${ }^{1}$ Neuro-imaging studies are also suggested. Diffusion-weighted Imaging, positron emission tomography and single photon emission computed tomography (CT) are preferable to computed tomography whose findings are usually negative during the attack.

However, head CT is usually the only diagnostic test available quickly in the Emergency Department (ED). In this context, investigations should also include laboratory tests to exclude intoxication, infection, and metabolic derangements. Patients with recurrent or brief episodes, or activity suggesting motor automatisms should be evaluated with electroencephalogram (EEG) for possible epilepsy. Despite this, an TGA episode has such specific character- 
istics that, when correctly framed, can be diagnosed only on clinical parameters with a good level of certainty. ${ }^{17}$

Treatment is not required for TGA, and the prognosis is generally benign. Recurrence rates range from 8 to 18 percent with variable follow-up. There is no increased risk of mortality, epilepsy, or stroke following TGA as compared with age-matched control subjects..$^{2,18}$

In this study, we evaluated the prognosis in patients presenting TGA clinical features in patients examined at the ED.

Objectives of the study were as follows: i) to verify the frequency of subsequent episodes of TGA in the cohort of patients examined; ii) to identify any predisposing factors for the occurrence of TGA; iii) to evaluate TGA as a possible predictor of neurological disease.

\section{Materials and Methods}

A cohort study was conducted at the ED of the University Hospital of Pisa on patients with TGA diagnosis (hospitalized, discharged or under observation patients) in the period from January 1, 2010 to December 31st, 2014, subjected to subsequent followup evaluation with a telephone interview.

\section{Study population}

A total of 119 patients were selected, 57 (47.90\%) women and 62 men $(52.10 \%)$, with a mean age of $64.02 \pm 9.06$ years. Among the 119 patients examined in the ED, $56(47.05 \%)$ were not included in the analysis: $44(36.97 \%)$ no traced by telephone; $10(8.40 \%)$ with no availability to telephone interview; 2 (1.68\%) deceased.

\section{Survey tools}

Survey tools included: clinical record of access to the emergency room (Table 1), obtained through FirstAid® program (Dedalus Heathcare System Group, Florence, Italy), in use at the ED of the hospital of Pisa, telephone questionnaires, based on literature; diagnostic criteria of Hodges and Warlow. ${ }^{1}$

\section{Telephone questionnaires}

In agreement with ischemic-thromboembolic TGA pathogenesis, ${ }^{9,10}$ we evaluated, the presence of previous cardiovascular (CV) events or main $\mathrm{CV}$ risk factors possibly associated with TGA in our patient cohort.

Patients were asked whether the TGA episode was the first, and, if it had not been, the number and the indicative date of the other previous episodes were asked, the presence of head trauma prior to TGA episode, current therapy, events precipitating the episode, presence of associated symptoms.
The second part of the questionnaire concerns the follow-up and in particular evaluated: i) additional episodes of TGA (annotations on these episodes and the indicative date of them); ii) acute cerebral events (ischemic or hemorrhagic stroke, transient ischemic attack, cerebral hemorrhage, epileptic seizures); iii) diagnosis of neurodegenerative disease (Parkinson's disease; Amyotrophic Lateral Sclerosis, ALS; Multiple Sclerosis, MS; Alzheimer's disease or other forms of dementia); iv) presence of a depressive state through the Geriatric Depression Scale (GDS) Short Form (15 Items); v) post-TGA functional autonomy: evaluation performed using the Basic Activities or Daily Living (BADL) and Instrumental Activities of Daily Living (IADL) scales.

\section{Hodges e Warlow criteria}

Hodges e Warlow criteria are as follows: ${ }^{1}$ i) witnessed event; ii) acute onset of anterograde amnesia with or without a mild retrograde amnesia; iii) no accompanying neurologic signs or symptoms (aphasia, apraxia or loss of personal identity); iv) no alteration of consciousness; v) no epileptic features; vi) resolution of symptoms within $24 \mathrm{~h}$; vii) other causes of amnesia excluded (recent head trauma or active epilepsy).

\section{Statistical analysis}

Data are expressed as mean \pm standard deviation for continuous numeric variables and as a frequency for categorical categories.

In light of the limited sample size, in our analysis we used the Fisher's chi square test for comparisons between variables expressed as frequencies. A significant difference was for $\mathrm{P}<0.05$.

\section{Results}

\section{Information on access to the Emergency Department}

We enrolled 63 patients; $33(52.31 \%)$ were males and 30 (47.69\%) females.

Mean age at the time the episode presentation was $64.55 \pm 8.66$ (61.69 \pm 8.84 years for men and $67.7 \pm 7.39$ years for women), being the younger and older patients 46 and 82 years, respectively. The most represented age group is between 65 and 75 years, with a total of 31 cases $(49.21 \%)$ (Table 2).

$C V$ risk factors, previous cardiac and cerebrovascular events, neurological disease and psychiatric disorder. Their prevalence is reported in Table 3.

Previous episodes of TGA. From the analysis of the medical records it was possible to identify patients who had one or more previous episodes of TGA. They were 9 (14.29\%), of which 5

Table 1. Medical record of access to the emergency room.

\begin{tabular}{ll}
\hline $\begin{array}{l}\text { Personal data } \\
\text { Past medical history }\end{array}$ & $\begin{array}{l}\text { Age, gender, date of access to ED, telephone number } \\
\text { Previous diseases, previous episodes of TGA, cardiovascular risk factors }\end{array}$ \\
\hline Recent medical history & Dynamics of the event, trigger factors, witnesses, associated symptoms \\
Pharmacological anamnesis & Anti-aggregating or anticoagulant therapy in progress \\
\hline Instrumental diagnostics & Head CT, Carotid and vertebral color flow guided duplex US, EEG \\
Neurological counseling & Indication to perform instrumental examination or drug therapy \\
\hline Discharge therapy & Antiplatelet, antiepileptic, psychiatric drugs \\
Outcome & Home discharge, voluntary discharge, observation, hospitalization \\
\hline ED, Emergency Department: TGA, transient global amnesia; CT, computed tomography: EEG, electroencephalogram.
\end{tabular}


$(55.55 \%)$ of females and $4(44.45 \%)$ of males.

Pharmacological treatment. None of our 63 patients at the time of the TGA were taking anticoagulant therapy and 15 $(23.81 \%)$ patients were taking antiplatelet therapy.

Triggering factors. 32 out of the 63 patients $(50.79 \%)$ manifested the TGA in a rest condition (absence of triggers, both physical and emotional). Among the other 31 (49.21\%) patients, 18 (28.57\%) manifested the TGA following a particular emotional stress, $4(6.35 \%)$ following an intense physical effort, $4(6.35 \%)$ following sexual intercourse, $3(4.76 \%)$ following a very hot shower or bath, $3(4.76 \%)$ following other triggering events and only one patient (1.59\%) developed the TGA following an invasive medical procedure (angiographic procedure). None of our patients reported, like possible trigger factor, driving a vehicle, a cold bath or an intense physical pain.

Head trauma in the 30 days prior to the event. Of the 63 patients interviewed, only $1(1.59 \%)$ reported a head trauma in the 30 days prior to the TGA episode.

Symptoms associated with the TGA episode. Of the 63 patients enrolled, 49 (77.78\%) did not show symptoms associated with the TGA. Regarding the 14 patients who reported such symptoms, 8 $(12.70 \%)$ cases reported headache, $3(4.76 \%)$ nausea, $3(4.76 \%)$ vomiting, $3(4.76 \%)$ patients reported other symptoms (buzzing, vertigo).

Diagnostic tests. All 63 patients underwent neurological counseling, which was normal except for memory lapses for the event and, when still present, an anterograde amnesia. All of the patients had a head CT scan, which demonstrated no acute lesions. Carotid and vertebral color flow guided duplex US was performed in 22 patients $(34.92 \%)$ with positive result for hemodynamic significant lesions in 2 patients $(3.17 \%)$.

Discharge therapy from the ED. 15 patients out of 63 (23.81\%) were on antiplatelet therapy and it was continued. In the 48 patients (76.19\%) who were not on antiplatelet therapy, it was recommended in 26 of them $(54.17 \%)$. Overall antiplatelet therapy was recommended in $41(65.08 \%)$ patients.

Level of urgency and outcome. A red code was assigned to 6 patients $(9.52 \%)$, yellow code to $43(68.25 \%)$ and green code to 14 $(22.22 \%)$. Of the aforementioned patients, 37 (58.73\%) were discharged at home, $19(30.16 \%)$ were subjected to observation, 7 $(11.11 \%)$ were hospitalized.

\section{Follow-up}

The average duration of follow-up was $40.23 \pm 16.59$ months (from 13 to 68 months).

Subsequent episodes of TGA. 6 patients, all males, showed subsequent episodes of TGA during the follow-up period (frequency of TGA recurrence $9.52 \%$ ). The average age was $61 \pm 7.82$ years. The most represented age group was between 45 and 55 years (50\%). Five patients $(83.34 \%)$ showed a single episode, and 1 patient $(16.66 \%)$ showed 2 . The average time elapsed between the first episode and subsequent one was $26.16 \pm 18.43$ months (range 11-61 months). We have analyzed the conditions that could predispose to a second TGA episode (male sex, previous episodes of TGA, history of migraine, CV risk factors including age, history of atrial fibrillation (AF), psychiatric pathology and antiplatelet therapy). No factor demonstrated a statistically significant correlation $(\mathrm{P}>0.05)$ with a second episode, except for male sex $\left(\mathrm{X}^{2}<0.05\right)$.

\section{Subsequent acute neurological events and diagnosis of neurodegenerative diseases}

During follow-up, 2 patients $(3.17 \%)$ developed one or more of the acute neurological events. Both of these patients were female. The average age is $69 \pm 0.7$ years (range $68-70$ years). One patient $(1.59 \%)$ subsequently had an epileptic seizure, the other had several episodes of epileptic seizures and then an ischemic stroke. The average time elapsed between these episodes and the TGA episode was $39 \pm 12.73$ months (range 30-48 months). None of the patients developed transient ischemic attack, brain hemorrhages or other acute neurological events during follow-up. The frequency of epileptic seizures in our sample of patients, during the follow-up, is therefore $3.17 \%$, while that of ischemic stroke of $1.59 \%$. None of the 63 patients had a diagnosis of a degenerative disease (Alzheimer's disease or other forms of dementia, Parkinson's disease, ALS, MS) at baseline and during follow-up.

Evaluation of the presence of depressive state through GDS15. All patients completed the questionnaire, answering the 15

Table 2. Frequency of transient global amnesia by age group.

\begin{tabular}{lcc} 
Age range (years) & Frequency $(\mathrm{n} / \mathrm{d}$ (63) & Rate $(\%)$ \\
$45-55$ & 10 & 15.87 \\
$55-65$ & 16 & 25.40 \\
\hline $65-75$ & 31 & 49.21 \\
$75-85$ & 6 & 9.52 \\
\hline
\end{tabular}

Table 3. Previous cardiovascular risk factors and events, neurological disease, psychiatric disorder in our population.

\begin{tabular}{lcc} 
Pre-existing disease & Frequency (n./63) & Rate (\%) \\
CV risk factors/events & & \\
Hypertension & 30 & 47.62 \\
Dyslipidemia & 27 & 42.62 \\
\hline Smoking & 20 & 31.75 \\
Family history of CV events & 14 & 22.22 \\
\hline Diabetes mellitus & 4 & 6.35 \\
CV events & 4 & 6.35 \\
\hline Atrial fibrillation & 4 & 6.35 \\
Neurological/psychiatric disease & \\
Migraine & 12 & 19.05 \\
Epilepsy & 0 & 0 \\
\hline Anxious syndrome & 8 & 12.7 \\
Depressive syndrome & 6 & 9.52 \\
\hline Panic attacks & 2 & 3.23 \\
Bipolar disorder & 1 & 1.61 \\
\hline CV, cardiovascular. & &
\end{tabular}

Table 4. Frequency of transient global amnesia from 2010 to 2015.

\begin{tabular}{lcc} 
Presentation year & Frequency $(\mathrm{n} / \mathbf{1} 19)$ & Rate (\%) \\
2010 & 19 & 15.97 \\
2011 & 29 & 24.37 \\
\hline 2012 & 22 & 18.48 \\
2013 & 31 & 26.05 \\
\hline 2014 & 18 & 15.13 \\
Total & 119 & 100 \\
\hline
\end{tabular}


questions under consideration. The average of the total score was $2.67 \pm 2.78$ (range $0-12$ ); a normal score (0-4) was found in 54 patients (85.71), a mild depressive state (score 5-10) was recorded in 8 patients $(12.70 \%)$ and only $1(1.59 \%)$ patient scored between 10 and 15 , an indicator of a severe depressive state. Overall, a depressive state was found in 9 patients (14.29\%), 5 females (frequency $12.12 \%$ ) and 4 males $(16.67 \%)$, respectively. The mean age of the group of patients with depression was $64.89 \pm 5.89$ years (range 57-73 years), similar to that observed whereas in patients without depressive state (64.50 $\$ 9.10$ years; range $46-82$ years).

Evaluation of functional autonomy. All 63 patients completed BADL and IADL scales. The average score for the BADL scale was $5.71 \pm 0.55$ (range 4-6 points). The average score for the IADL scale was 7.53 points \pm 0.72 (range $5-8$ points). Only 2 (3.28\%) out of 63 patients were non-autonomous at the follow-up assessment.

\section{Discussion}

In our study, the incidence of the TGA is concordant with that reported by Zeman and Hodges in $1997 .{ }^{19}$ On a population of 421,816 inhabitants (according to ISTAT data), Pisa area average incidence of TGA is around 5.64 cases per 100,000 inhabitants per year (Table 4). The mean age (64 years) was in line with that reported in comparison studies. ${ }^{1,2,6,20}$

We have assessed both by clinical record analysis and telephone interview, whether the diagnosis had been made according to Hodges and Warlow criteria. ${ }^{1}$ Recent trauma and active epilepsy were excluded, and no focal neurologic symptoms were reported in $98 \%$ of the patients. The attack had been witnessed in $95 \%$ of the cases.

Regarding the prevalence of CV risk factors in our population, we did not find a significantly difference compared to general population, in agreement with an Italian study conducted in $2012^{21}$ and other studies from literature. . $^{2,18,22-24}$ This confirms a similar prevalence of $\mathrm{CV}$ risk factor in TGA and not TGA patients.

Concerning co-morbidities, the prevalence of AF is higher than that reported by Zorzon et al. ${ }^{20}$ but is even lower than that reported in the study by Pantoni et al. ${ }^{6}$ The prevalence of migraine, epilepsy and psychiatric disorder are in line with the literature. , $, 2,20^{2}$

The most reported triggering factors found in our study were emotional stress, in line with what was reported by Quinette et al. ${ }^{2}$ Regarding the other factors taken into consideration, we found results which are fairly in line with the literature, except for the frequency of physical exercise as triggering event, since it was lower in our sample (6\%) as compared to that described in the previous studies. ${ }^{2,6,15}$

During the follow-up, TGA episodes recurrence was $9.52 \%$ (6 patients, $100 \%$ of whom were male aged 55 to 65 years). Our results are in line with the previous studies $, 6,20$ in which the recurrence rate is on average $9 \%$. It has to be noted that the higher recurrence rates have been obtained in studies in which probable cases were also included.

Among conditions that could predispose to a second TGA episode (male sex, previous episodes of TGA, history of migraine, $\mathrm{CV}$ risk factors, history of $\mathrm{AF}$, psychiatric pathology and antiplatelet therapy) only male sex was associated with TGA recurrence in our series. This result suggests that the male sex predisposes to the recurrence of TGA, despite the limited sample size does not strongly support this conclusion. However, also analyzing the history at ED's admission, we observed a prevalence of males (66\%), as compared to females within the 9 patients with previous
TGA episodes. Age between 65 and 75 years is also not significantly associated with TGA recurrence.

From the information obtained at follow-up, we observed that $14 \%$ of patients had a GDS-15 diagnostic score for depression, and there were acute neurological events in 2 patients, while none developed a neurodegenerative disease. Also, in this case the results are in agreement with those of the literature..$^{6,20,25}$

Most of our patients were autonomous from the functional point of view according to IADL e BADL scales.

In summary, our results confirm that TGA is more represented in the male sex; the disease is recurrent in about $10 \%$ of patients, and this recurrence is greater in the male sex, TGA is not a predictor of neurological disease.

Important observation emerged for the TGA clinical management within the ED. Most of the patients were discharged home. Approximately $30 \%$ of the patients were retained in observation unit, as the symptoms were not yet resolved, and all of them were discharged within 24 hours without evidence of clinical development of the symptoms.

Among the 7 patients admitted for further evaluation according the clinical history and co-morbidities, all of them were discharged, asymptomatic and in the absence of other clinical manifestations.

The analysis of the 2 cases showing an acute neurological event (epilepsy) in the following years, demonstrated that both were female. In one of the cases the diagnosis of TGA did not comply with all the diagnostic criteria (attack not witnessed) and in both cases an EEG had not been performed following indication

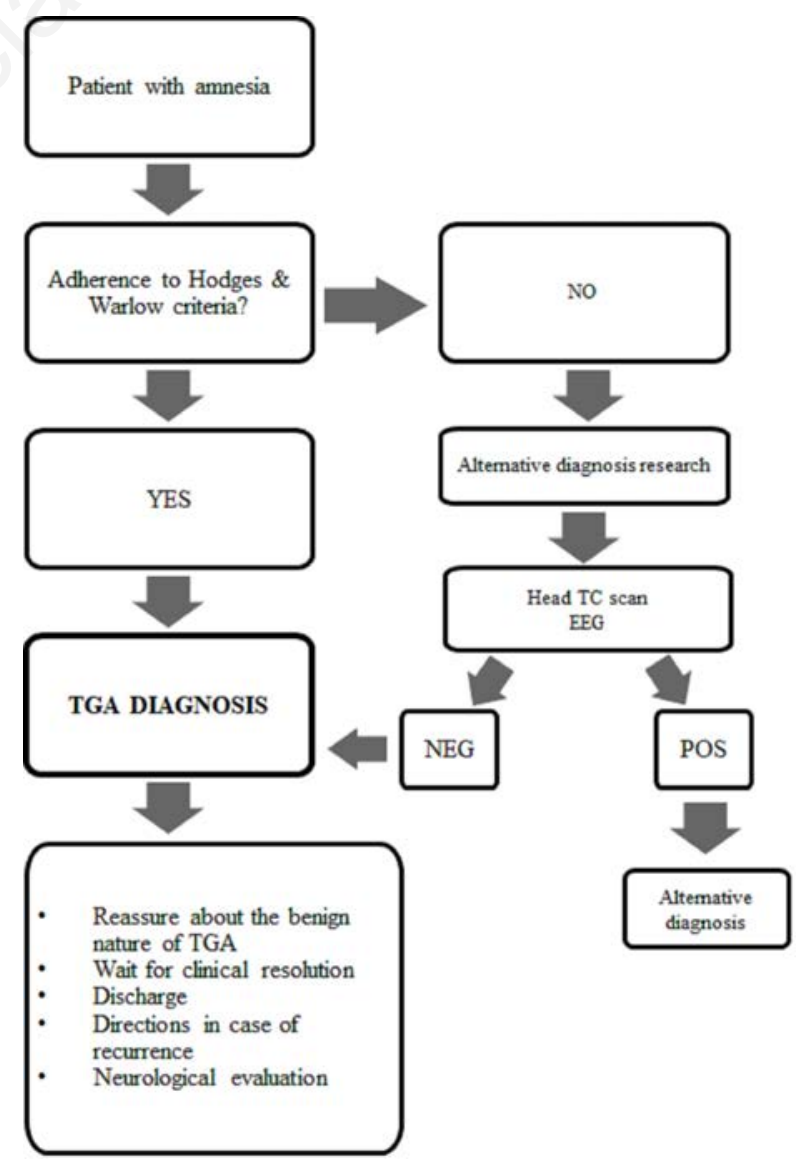

Figure 1. Transient global amnesia (TGA) diagnostic flow-chart in the Emergency Department. 
related to TGA. It is therefore likely that the diagnosis of TGA in these two cases could have been erroneously placed at first admissions, since an episode of epilepsy was not excluded. These data underline the importance of carrying out an EEG in order to perform a correct differential diagnosis in patients where Hodges and Warlow's criteria are not fully respected and therefore TGA diagnosis is not completely supported.

The strength of the present study is represented by the followup for evaluating TGA recurrence. However, the study has some limitations to be acknowledged. The main limitations are represented by the retrospective selection of patients, the relative low sample size and the lack of control group. Furthermore, the percentage of dropouts from the study is considerably high (almost a half of the study population) since patients are not traceable by telephone or not provide consent to the telephone questionnaire and 2 patients $(2 \%)$ were not included in the follow-up evaluation because they died. This may have influenced the results. In fact, the causes of death of deceased patients have not been precisely verified and patients who have refused to participate in the telephone interview may also have developed significant health problems.

\section{Conclusions}

Our study confirms that TGA is a benign pathology with a low probability of relapse. Its correct initial clinical classification, with full respect of the Hodges and Warlow criteria, would allow the patient to be rapidly resigned at the clinical resolution even in the ED setting.

A diagnostic procedure is suggested for a simpler and faster management of patients with TGA in ED (Figure 1), on the basis of the literature and the results of the study. It underlines the importance of a correct initial clinical classification (gender, age, Hodges and Warlow). If one or more diagnostic criteria are not met, an alternative diagnosis must be evaluated using second-level instrumental tests (head CT scan and EEG) and possibly a specialist neurological evaluation. After being diagnosed with TGA, the patient can be discharged with a certainty of diagnosis and reassured of the benign nature of the disease, but with the recommendation to return to ED if the disorder is repeated to perform an elective neurological examination within 10 days.

\section{References}

1. Hodges JR, Warlow CP. Syndromes of transient amnesia: towards a classification. A study of 153 cases. J Neurol Neurosurg Psychiatry 1990;53:834-43.

2. Quinette P, Guillery-Girard B, Dayan J, et al. What does transient global amnesia really mean? Review of the literature and thorough study of 142 cases. Brain 2006;129:1640-58.

3. Arena JE, Rabinstein AA. Transient global amnesia. Mayo Clin Proc 2015;90:264-72.

4. Matias-Guiu J, Blanquer J, Falip R, et al. Incidence of transient global amnesia in Alcoi (Spain). Acta Neurol Scand 1992;86:221.

5. Lauria G, Gentile M, Fassetta G, et al. Incidence of transient global amnesia in the Belluno province, Italy: 1985 through 1995. Results of a community-based study. Acta Neurol Scand
1997;95:303-10.

6. Pantoni L, Bertini E, Lamassa M, et al. Clinical features, risk factors, and prognosis in transient global amnesia: a follow-up study. Eur J Neurol 2005;12:350-6.

7. Owen D, Paranandi B, Sivakumar R, Seevaratnam M. Classical diseases revisited: transient global amnesia. Postgrad Med J 2007;83:236-9.

8. Maalikjy Akkawi N, Agosti C, Anzola GP, et al. Transient global amnesia: a clinical and sonographic study. Eur Neurol 2003;49:67-71.

9. Felix MM, Castro LH, Maia AC, Jr., da Rocha AJ. Evidence of acute ischemic tissue change in transient global amnesia in magnetic resonance imaging: case report and literature review. J Neuroimaging 2005;15:203-5.

10. Di Filippo M, Calabresi P. Ischemic bilateral hippocampal dysfunction during transient global amnesia. Neurology 2007;69:493.

11. Kapur N. Transient epileptic amnesia a clinical update and a reformulation. J Neurol Neurosurg Psychiatry 1993;56:1184-90.

12. Bergerot A, Holland PR, Akerman S, et al. Animal models of migraine: looking at the component parts of a complex disorder. Eur J Neurosci 2006;24:1517-34.

13. Sander K, Sander D. New insights into transient global amnesia: recent imaging and clinical findings. Lancet Neurol 2005;4:437.

14. Jang JW, Park SY, Hong JH, et al. Different risk factor profiles between transient global amnesia and transient ischemic attack: a large case-control study. Eur Neurol 2014;71:19.

15. Inzitari D, Pantoni L, Lamassa M, et al. Emotional arousal and phobia in transient global amnesia. Arch Neurol 1997;54:866-73.

16. Kessler J, Markowitsch HJ, Rudolf J, Heiss WD. Continuing cognitive impairment after isolated transient global amnesia. Int $\mathbf{J}$ Neurosci 2001;106:159-68.

17. Brown J. ED evaluation of transient global amnesia. Ann Emerg Med 1997;30:522-6.

18. Miller JW, Petersen RC, Metter EJ, et al. Transient global amnesia - clinical characteristics and prognosis. Neurology 1987;37:733-7.

19. Zeman AZ, Hodges JR. Transient global amnesia. Br J Hosp Med 1997;58:257-60.

20. Zorzon M, Antonutti L, Mase G, et al. Transient global amnesia and transient ischemic attack: natural history, vascular risk factors, and associated conditions. Stroke 1995;26:1536-42.

21. Tragni E, Filippi A, Casula M, et al. Risk factors distribution and cardiovascular disease prevalence in the Italian population: The CHECK study. Open J Epidemiol 2012;2:90-100.

22. Nausieda P, Sherman I. Long-term prognosis in transient global amnesia. JAMA 1979;241:392-3.

23. Crowell G, Stump D, Biller J, et al. The transient global amnesiamigraine connection. Arch Neurol 1984;41:75-9.

24. Hinge H-H, Jensen T, Kjaer M, et al. The prognosis of transient global amnesia: results of a multicenter study. Arch Neurol 1986;43:673-6.

25. Hodges JR, Warlow CP. The etiology of transient global amnesia: a case-control study of 114 cases with prospective follow-up. Brain 1990;113:639-57. 